Virtual Special Issue - In memory of Professor Sibe Mardesic

\title{
Dissipative flows, global attractors and shape theory ${ }^{\text {is }}$
}

\author{
Héctor Barge $^{\mathrm{a}}$, José M.R. Sanjurjo ${ }^{\mathrm{b}, *}$ \\ a ETSI Informáticos, Universidad Politécnica de Madrid, Boadilla del Monte 28660, Madrid, Spain \\ b Facultad de C. C. Matemáticas, Universidad Complutense de Madrid, Madrid 28040, Spain
}

\section{A R T I C L E I N F O}

\section{Article history:}

Received 21 December 2016

Accepted 30 October 2017

Available online 19 March 2019

Dedicated to the memory of Sibe Mardešić

\section{$M S C$ :}

$34 \mathrm{D} 23$

$54 \mathrm{H} 20$

$55 \mathrm{P} 55$

$34 \mathrm{D} 10$

$37 \mathrm{C} 70$

$37 \mathrm{~B} 30$

\section{Keywords:}

Dissipative flows

Shape

Alexandrov compactification

Conley index

Continuation

Bifurcation

\begin{abstract}
A B S T R A C T
In this paper we study continuous parametrized families of dissipative flows, which are those flows having a global attractor. The main motivation for this study comes from the observation that, in general, global attractors are not robust, in the sense that small perturbations of the flow can destroy their globality. We give a necessary and sufficient condition for a global attractor to be continued to a global attractor. We also study, using shape theoretical methods and the Conley index, the bifurcation global to non-global.
\end{abstract}

(C) 2019 Elsevier B.V. All rights reserved.

\section{Introduction}

This paper is devoted to the study of continuous parametrized families of dissipative flows $\varphi_{\lambda}: M \times \mathbb{R} \rightarrow$ $M$, where $\lambda \in[0,1]$ and $M$ is a non-compact, locally compact metric space.

Dissipative flows have been introduced by Levinson [18] and they are a very important class of flows since, as it has been proved by Pliss [24], they agree with those flows having a global attractor. We provide a proof of this fact for the sake of completeness (see Proposition 2). Dissipative flows play a crucial role in

\footnotetext{
The authors are supported by MINECO (MTM2015- 63612-P).

* Corresponding author.

E-mail addresses: h.barge@upm.es (H. Barge), jose_sanjurjo@mat.ucm.es (J.M.R. Sanjurjo).
} 
mathematical ecology since they model the limitations of the environment (see for example $[3,11,16,34]$ ). An interesting reference regarding dissipative flows is [14].

The main motivation for this paper comes from the observation, pointed out in Example 5, that, in general, global attractors are not robust, in the sense that small perturbations of the flow can destroy their globality.

In section 1 we recall the definition of dissipative flow and we prove, for the sake of completeness, that a flow is dissipative if and only if it possesses a global attractor (Proposition 2). We also see in Example 5 that a small perturbation of a global attractor can produce a non-global attractor. Moreover, we see that in fact, it can have a bounded region of attraction. This fact motivates the definition of coercive family of dissipative flows. In addition, we introduce the concept of uniformly dissipative family of flows and we prove Theorem 9 which establishes that for a family of dissipative flows a global attractor continues to global attractors if and only if the family is uniformly dissipative. We illustrate this result by showing that in the Lorenz equations the global attractor continues to global attractors. We finish this section by proving Theorem 11, in which a topological study of coercive families of dissipative flows in $\mathbb{R}^{n}$ is made. In particular we see that if a parametrized family of dissipative flows is coercive then, given a continuation $K_{\lambda}$ of the global attractor $K_{0}$ of $\varphi_{0}$, an isolated invariant compactum $C_{\lambda} \subset \mathbb{R}^{n} \backslash K_{\lambda}$ with the shape of $S^{n-1}$ which attracts uniformly all the points of the unbounded component of its complement and repels all the points of the bounded one which are not in $K_{\lambda}$ is created for all $\lambda>0$ small. Besides, we see that the diameter of $C_{\lambda}$ goes to infinity when $\lambda$ goes to zero. Moreover, we see that the existence of such a $C_{\lambda}$ for all $\lambda>0$ small is sufficient for the family to be coercive.

In section 2 we study in all its generality the mechanism which produces the bifurcation global to non-global in families of dissipative flows. With this aim we introduce the definition of polar family of dissipative flows and we see (Proposition 14) that a family of dissipative flows is polar if and only if every continuation $K_{\lambda}$ of the global attractor $K_{0}$ of $\varphi_{0}$ satisfies that $K_{\lambda}$ is not a global attractor for all $\lambda>0$ small. In addition we prove Theorem 15 which establishes that if a parametrized family of dissipative flows is polar then, given a continuation $K_{\lambda}$ of the global attractor $K_{0}$ of $\varphi_{0}$, for all $\lambda>0$ small the maximal invariant compactum $C_{\lambda} \subset \mathbb{R}^{n} \backslash K_{\lambda}$ is non-empty, isolated and has trivial cohomological Conley index.

Through the paper we often consider the Alexandrov compactification $M \cup\{\infty\}$ and we extend the family of flows $\varphi_{\lambda}: M \times \mathbb{R} \rightarrow M$ to a family of flows, which we also denote by $\varphi_{\lambda}$, on $M \cup\{\infty\}$ by leaving $\infty$ fixed for all $\lambda \in[0,1]$.

A form of homotopy theory, namely shape theory, which is the most suitable for the study of global topological properties in dynamics, will be occasionally used. Although a deep knowledge of shape theory is not necessary to understand the paper we recommend to the reader Borsuk's monography [2] and the books by Mardešić and Segal [20] and Dydak and Segal [8] for an exhaustive treatment of the subject and $[17,25,26,30-33,29,13,12]$ for a concise introduction and some applications to dynamical systems.

We make use of some notions of algebraic topology. Good references for this material are the books of Hatcher and Spanier $[15,35]$. We will use the notation $H_{*}$ and $H^{*}$ for the singular homology and cohomology with integer coefficients respectively.

The main reference for the elementary concepts of dynamical systems will be [1] but we also recommend $[27,22,23]$. By the omega-limit of a point $x$ we understand the set $\omega(x)=\bigcap_{t>0} \overline{x[t, \infty)}$ while the negative omega-limit is the set $\omega^{*}(x)=\bigcap_{t<0} \overline{x(-\infty, t]}$. An invariant compactum $K$ is stable if every neighborhood $U$ of $K$ contains a neighborhood $V$ of $K$ such that $V[0, \infty) \subset U$. Similarly, $K$ is negatively stable if every neighborhood $U$ of $K$ contains a neighborhood $V$ of $K$ such that $V(-\infty, 0] \subset U$. The compact invariant set $K$ is said to be attracting provided that there exists a neighborhood $U$ of $K$ such that $\emptyset \neq \omega(x) \subset K$ for every $x \in U$ and repelling if there exists a neighborhood $U$ of $K$ such that $\emptyset \neq \omega^{*}(x) \subset K$ for every $x \in U$. An attractor (or asymptotically stable compactum) is an attracting stable set and a repeller is a repelling negatively stable set. We stress the fact that stability (positive or negative) is required in the definition of attractor or repeller. If $K$ is an attractor, its region (or basin) of attraction $\mathcal{A}(K)$ is the set of all points 
$x \in M$ such that $\emptyset \neq \omega(x) \subset K$. It is well known that $\mathcal{A}(K)$ is an open invariant set. The region of repulsion of a repeller $K, \mathcal{R}(K)$ is defined in a dual way. If in particular $\mathcal{A}(K)$ is the whole phase space we say that $K$ is a global attractor.

Notice that, since we will deal with parametrized families of flows we will use the notation $\omega_{\lambda}(x), \omega_{\lambda}^{*}(x)$, $\mathcal{A}_{\lambda}(K)$ and $\mathcal{R}_{\lambda}(K)$ to denote the omega-limit, negative omega-limit, region of attraction and region of repulsion with respect to the flow $\varphi_{\lambda}$.

There is a nice topological relation between an attractor $K$ and its region of attraction $\mathcal{A}(K)$ expressed in terms of shape theory. It establishes that the inclusion $i: K \hookrightarrow \mathcal{A}(K)$ a shape equivalence [17, Theorem 3.6]. As a consequence, if $K$ is a global attractor of a flow in an Euclidean space it must have the shape of a point. In particular, it must be connected.

An important class of invariant compacta is the so-called isolated invariant sets (see [4,5,9] for details). These are compact invariant sets $K$ which possess an isolating neighborhood, i.e. a compact neighborhood $N$ such that $K$ is the maximal invariant set in $N$.

A special kind of isolating neighborhoods will be useful in the sequel, the so-called isolating blocks, which have good topological properties. More precisely, an isolating block $N$ is an isolating neighborhood such that there are compact sets $N^{i}, N^{o} \subset \partial N$, called the entrance and the exit sets, satisfying

1. $\partial N=N^{i} \cup N^{o}$;

2. for each $x \in N^{i}$ there exists $\varepsilon>0$ such that $x[-\varepsilon, 0) \subset M-N$ and for each $x \in N^{o}$ there exists $\delta>0$ such that $x(0, \delta] \subset M-N$;

3. for each $x \in \partial N-N^{i}$ there exists $\varepsilon>0$ such that $x[-\varepsilon, 0) \subset \stackrel{\circ}{N}$ and for every $x \in \partial N-N^{o}$ there exists $\delta>0$ such that $x(0, \delta] \subset \stackrel{\circ}{N}$.

These blocks form a neighborhood basis of $K$ in $M$.

Let $K$ be an isolated invariant set. Its Conley index $h(K)$ is defined as the pointed homotopy type of the topological space $\left(N / N^{o},\left[N^{o}\right]\right)$, where $N$ is an isolating block of $K$. A weak version of the Conley index which will be useful for us is the cohomological index defined as $C H^{*}(K)=H^{*}(h(K))$. The homological index $C H_{*}(K)$ is defined in an analogous fashion using homology. Notice that in fact, $C H^{*}(K) \cong H^{*}\left(N, N^{o}\right)$ and $C H_{*}(K) \cong H_{*}\left(N, N^{o}\right)$. Our main references for the Conley index theory are $[4,6,28]$.

In this paper the concept of continuation of isolated invariant sets plays a crucial role. Let $M$ be a locally compact metric space, and let $\varphi_{\lambda}: M \times \mathbb{R} \rightarrow M$ be a parametrized family of flows (parametrized by $\lambda \in[0,1]$, the unit interval). The family $\left(K_{\lambda}\right)_{\lambda \in J}$, where $J \subset[0,1]$ is a closed (non-degenerate) subinterval and, for each $\lambda \in J, K_{\lambda}$ is an isolated invariant set for $\varphi_{\lambda}$ is said to be a continuation if for each $\lambda_{0} \in J$ and each $N_{\lambda_{0}}$ isolating neighborhood for $K_{\lambda_{0}}$, there exists $\delta>0$ such that $N_{\lambda_{0}}$ is an isolating neighborhood for $K_{\lambda}$ for every $\lambda \in\left(\lambda_{0}-\delta, \lambda_{0}+\delta\right) \cap J$. We say that the family $\left(K_{\lambda}\right)_{\lambda \in J}$ is a continuation of $K_{\lambda_{0}}$ for each $\lambda_{0} \in J$.

Notice that [28, Lemma 6.1] ensures that if $K_{\lambda_{0}}$ is an isolated invariant set for $\varphi_{\lambda_{0}}$, there always exists a continuation $\left(K_{\lambda}\right)_{\lambda \in J_{\lambda_{0}}}$ of $K_{\lambda_{0}}$ for some closed (non-degenerate) subinterval $\lambda_{0} \in J_{\lambda_{0}} \subset[0,1]$.

There is a simpler definition of continuation based on [28, Lemma 6.2]. There, it is proved that if $\varphi_{\lambda}$ : $M \times \mathbb{R} \rightarrow M$ is a parametrized family of flows and if $N_{1}$ and $N_{2}$ are isolating neighborhoods of the same isolated invariant set for $\varphi_{\lambda_{0}}$, then there exists $\delta>0$ such that $N_{1}$ and $N_{2}$ are isolating neighborhoods for $\varphi_{\lambda}$, for every $\lambda \in\left(\lambda_{0}-\delta, \lambda_{0}+\delta\right) \cap[0,1]$, with the property that, for every $\lambda$, the isolated invariant subsets in $N_{1}$ and $N_{2}$ which have $N_{1}$ and $N_{2}$ as isolating neighborhoods agree.

Therefore, the family $\left(K_{\lambda}\right)_{\lambda \in J}$, with $K_{\lambda}$ an isolated invariant set for $\varphi_{\lambda}$, is a continuation if for every $\lambda_{0} \in J$ there are an isolating neighborhood $N_{\lambda_{0}}$ for $K_{\lambda_{0}}$ and a $\delta>0$ such that $N_{\lambda_{0}}$ is an isolating neighborhood for $K_{\lambda}$, for every $\lambda \in\left(\lambda_{0}-\delta, \lambda_{0}+\delta\right) \cap J$. 
We will make use of the fact that if $\left(K_{\lambda}\right)_{\lambda \in J}$ is a continuation then, for each $\lambda_{1}, \lambda_{2} \in J$, the Conley indices $h\left(K_{\lambda_{1}}\right)$ and $h\left(K_{\lambda_{2}}\right)$ agree (see [28, Corollary 6.8]). A consequence of this fact is that if $K_{\lambda_{0}}$ is a non-empty attractor and $\left(K_{\lambda}\right)_{\lambda \in J}$ is a continuation of it, then $K_{\lambda}$ is non-empty for each $\lambda \in J$.

We are interested in continuations $\left(K_{\lambda}\right)_{\lambda \in J}$, with $0 \in J$, where $K_{0}$ is a global attractor. Since $K_{0}$ is an attractor, using [32, Theorem 4] it follows that there exists $0<\lambda_{0} \in J$ such that, for $\lambda<\lambda_{0}, K_{\lambda}$ is an attractor which has the shape of $K_{0}$. As a consequence, if the phase space is a Euclidean space, then for small values of $\lambda, K_{\lambda}$ has the shape of a point and, in particular, it is connected.

Notice that, since this should not lead to any confusion, sometimes we will only say that $K_{\lambda}$ is a continuation of $K_{\lambda_{0}}$ without specifying the subinterval $J \subset[0,1]$ to which the parameters belong.

\section{Continuation of global attractors}

In this section we study parametrized families of dissipative flows and continuations of global attractors. In particular, we will see that a small perturbation of a global attractor can be a non-global attractor since, in fact, it can have a bounded region of attraction. We will also give a necessary and sufficient condition for the family $\left(K_{\lambda}\right)_{\lambda \in[0,1]}$ where, for each $\lambda \in[0,1] K_{\lambda}$ is the global attractor of $\varphi_{\lambda}$, to be a continuation of the global attractor $K_{0}$ of $\varphi_{0}$. Besides all this we give a descriptive characterization of coercive families of dissipative flows.

Definition 1 (Levinson 1944). We say that a flow $\varphi: M \times \mathbb{R} \rightarrow M$ is dissipative provided that for each $x \in M, \omega(x) \neq \emptyset$ and the closure of the set

$$
\Omega(\varphi)=\bigcup_{x \in M} \omega(x)
$$

is compact.

Proposition 2 (Pliss 1966). A flow $\varphi: M \times \mathbb{R} \rightarrow M$ is dissipative if and only if there exists a global attractor.

Proof. If $\varphi$ has a global attractor $K$, then it is clear that $\Omega(\varphi) \subset K$ and hence its closure is compact ensuring the dissipativeness of $\varphi$.

Conversely, suppose that $\varphi$ is dissipative. We extend the flow to the Alexandrov compactification $M \cup\{\infty\}$ leaving $\infty$ fixed. Let $N$ be a compact neighborhood of $\overline{\Omega(\varphi)}$. Then, $U=(M \cup\{\infty\}) \backslash N$ is a neighborhood of $\{\infty\}$ and, given any point $x \in M \backslash N=U \backslash\{\infty\}$, since $\emptyset \neq \omega(x) \subset N$, there exists a time $t_{x}>0$ such that $x t_{x} \notin U$. Hence, by [28, Lemma 3.1] $\{\infty\}$ is a repeller.

Since $M \cup\{\infty\}$ is compact and $\{\infty\}$ is a repeller, there exists a non-empty attractor $K \subset M$ dual to $\{\infty\}$. It is clear that this attractor $K$ must be a global attractor.

Remark 3. The dissipativeness of $\varphi$ is equivalent to $\{\infty\}$ being a repeller.

An easy consequence of these considerations is the following proposition.

Proposition 4. Let $\varphi$ be a dissipative flow in $\mathbb{R}^{n}$ and $K$ an attractor of $\varphi$. Then $K$ is global if and only if $\mathcal{A}(K) \backslash K$ does not contain bounded orbits.

Hence, if $K$ is an attractor of a dissipative flow, $\mathcal{A}(K)$ being bounded is in sharpest contrast to $K$ being global. 

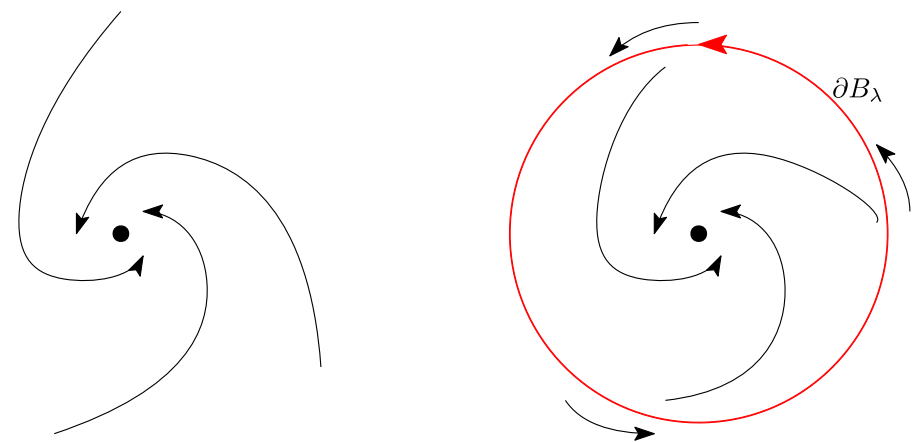

Fig. 1. Phase portrait of the family of equations (1). (For interpretation of the colors in the figure(s), the reader is referred to the web version of this article.)

Example 5. Consider the family of ordinary differential equations defined on the plane in polar coordinates

$$
\left\{\begin{array}{l}
\dot{r}=-r^{3}\left(\frac{1}{r}-\lambda\right)^{2}, \quad \lambda \in[0,1] \\
\dot{\theta}=1
\end{array}\right.
$$

The picture on the left in Fig. 1 describes the phase portrait of equation (1) when the parameter $\lambda=0$. We see that in this case the origin is a globally attracting fixed point and the orbit of any other point spirals towards it. The picture on the right describes the phase portrait of equation (1) when $\lambda>0$. In this case we see that the origin is still an asymptotically stable fixed point but it is not a global attractor anymore since, for each $\lambda>0$, the circle centered at the origin and radius $1 / \lambda$ is a periodic trajectory which attracts uniformly all the points of the unbounded component of its complement and repels all the points of the bounded one except the origin. Notice that for every $\lambda>0$ the disk $B_{\lambda}$ centered at the origin and radius $1 / \lambda$ is a global attractor whose interior agrees with the basin of attraction of $\{0\}$. As a consequence of these remarks, for each $\lambda \in[0,1]$ the flow $\varphi_{\lambda}$ associated to the corresponding value of $\lambda$ in (1) is dissipative and, if $K_{\lambda}$ is a continuation of the global attractor $\{0\}$ of $\varphi_{0}$, there exists $\lambda_{0}>0$ such that for each $0<\lambda<\lambda_{0}, K_{\lambda}=\{0\}$. Therefore, the global attractor $\{0\}$ cannot be continued to a family of global attractors.

Example 5 is an instance of parametrized family $\varphi_{\lambda}$ of dissipative flows in $\mathbb{R}^{2}$ with a global attractor $K_{0}$ such that every continuation $K_{\lambda}$ of $K_{0}$ satisfies that there exists $\lambda_{0}>0$ such that $\mathcal{A}_{\lambda}\left(K_{\lambda}\right)$ is bounded for every $0<\lambda<\lambda_{0}$. This motivates the following definition.

Definition 6. A parametrized family of dissipative flows $\varphi: M \times \mathbb{R} \rightarrow M$ is said to be coercive if for any continuation $K_{\lambda}$ of the global attractor $K_{0}$ of $\varphi_{0}$, there exists $\lambda_{0}>0$ such that $\mathcal{A}_{\lambda}\left(K_{\lambda}\right)$ is bounded for every $0<\lambda<\lambda_{0}$.

Remark 7. Notice that it follows from [28, Lemma 6.2] that if $K_{0}$ has a continuation $K_{\lambda}$ satisfying that there exists $\lambda_{0}>0$ such that $\mathcal{A}_{\lambda}\left(K_{\lambda}\right)$ is bounded for every $0<\lambda<\lambda_{0}$, then the family $\varphi_{\lambda}$ is coercive.

Before studying coercive families of dissipative flows we will characterize when a global attractor can be continued to a family of global attractors. For this purpose we introduce the following definition.

Definition 8. A parametrized family of flows $\varphi_{\lambda}: M \times \mathbb{R} \rightarrow M$ is said to be uniformly dissipative provided that $\omega_{\lambda}(x) \neq \emptyset$ for each $x \in M$ and $\lambda \in[0,1]$ and the closure of 


$$
\Omega=\bigcup_{\lambda \in[0,1]} \Omega\left(\varphi_{\lambda}\right)
$$

is compact.

Theorem 9. Let $\varphi_{\lambda}: M \times \mathbb{R} \rightarrow M$ be a parametrized family of dissipative flows with $\lambda \in[0,1]$. Suppose that $K_{\lambda}$ denotes the global attractor of $\varphi_{\lambda}$. Then, the family $\left(K_{\lambda}\right)_{\lambda \in[0,1]}$ is a continuation of $K_{0}$ if and only if the family $\left(\varphi_{\lambda}\right)_{\lambda \in[0,1]}$ is uniformly dissipative.

Proof. Suppose that the family of global attractors $\left(K_{\lambda}\right)_{\lambda \in[0,1]}$ is a continuation of the global attractor $K_{0}$ of $\varphi_{0}$. Then, for each $\lambda_{0} \in[0,1]$ there exists an isolating neighborhood $N_{\lambda_{0}}$ of $K_{\lambda_{0}}$ and $\delta>0$ such that $N_{\lambda_{0}}$ is an isolating neighborhood of $K_{\lambda}$ for $\lambda \in\left(\lambda_{0}-\delta, \lambda_{0}+\delta\right) \cap[0,1]$.

Using this fact and the compactness of $[0,1]$ it follows that there exist $\lambda_{1}, \ldots, \lambda_{k} \in[0,1]$ such that, if we call $K=\cup_{\lambda \in[0,1]} K_{\lambda}$,

$$
\bar{K} \subset \bigcup_{i=1}^{k} N_{\lambda_{k}} .
$$

As a consequence, $\bar{K}$ is compact. Moreover, $\omega_{\lambda}(x) \neq \emptyset$ for each $\lambda \in[0,1]$ and $x \in M$ since every flow in the family is dissipative and the closure of $\Omega$ is compact being contained in $\bar{K}$. Therefore, the family $\left(\varphi_{\lambda}\right)_{\lambda \in[0,1]}$ is uniformly dissipative.

Conversely, suppose that the family $\left(\varphi_{\lambda}\right)_{\lambda \in[0,1]}$ is uniformly dissipative. We see that the family of global attractors $\left(K_{\lambda}\right)_{\lambda \in[0,1]}$ is a continuation of the global attractor $K_{0}$. For this purpose we consider the family of flows extended to the Alexandrov compactification $M \cup\{\infty\}$ leaving $\infty$ fixed. Let $\lambda_{0} \in[0,1], N_{\lambda_{0}}$ an isolating neighborhood of $K_{\lambda_{0}}$ and $W$ a compact neighborhood of $N_{\lambda_{0}} \cup \Omega$. Then, $U=\overline{(M \cup\{\infty\}) \backslash W}$ is an isolating neighborhood of $\{\infty\}$ for the flow $\varphi_{\lambda_{0}}$. Since $\left(K_{\lambda_{0}},\{\infty\}\right)$ is an attractor-repeller decomposition, it follows from [28, Lemma 6.4] that there exists $\delta>0$ such that for each $\lambda \in\left(\lambda_{0}-\delta, \lambda_{0}+\delta\right) \cap[0,1]$ the pair $\left(A_{\lambda}, R_{\lambda}\right)$, where $A_{\lambda}$ is the isolated invariant set having $N_{\lambda_{0}}$ as isolating neighborhood and $R_{\lambda}$ is the maximal invariant set in $W$ for $\lambda \in\left(\lambda_{0}-\delta, \lambda_{0}+\delta\right) \cap[0,1]$, is an attractor-repeller decomposition. Besides, from the choice of $W$ it easily follows that $R_{\lambda}=\{\infty\}$ and, hence, $A_{\lambda}$ must agree with the global attractor $K_{\lambda}$ for $\lambda \in\left(\lambda_{0}-\delta, \lambda_{0}+\delta\right) \cap[0,1]$. Thus the result follows.

Example 10. An important example of a global attractor is provided by the Lorenz equations

$$
\left\{\begin{array}{l}
\dot{x}=\sigma(y-x) \\
\dot{y}=r x-y-x z \\
\dot{z}=x y-b z
\end{array}\right.
$$

where $\sigma, r$ and $b$ are three real positive parameters. E.N. Lorenz proved in [19] that for every value of $\sigma, r$ and $b$ there exists a global attractor of zero volume for the flow associated to these equations. This attractor should not be confused with the famous Lorenz attractor, which is a proper subset of the global attractor.

If we fix $\sigma$ and $b$ we obtain a family of flows $\varphi_{r}: \mathbb{R}^{3} \times \mathbb{R} \rightarrow \mathbb{R}^{3}$ corresponding to the Lorenz equations for the different values of $r$. Each of these flows has a global attractor $K_{r}$. We shall show that the family $\varphi_{r}$ is uniformly dissipative and, hence, for a given $r_{0}$ the family of global attractors $K_{r}$ is a continuation of $K_{r_{0}}$.

Sparrow studied in [36] the function

$$
V=r x^{2}+\sigma y^{2}+\sigma(z-2 r)^{2}
$$


which is a Lyapunov function for the flow $\varphi_{r}$. By using this function he was able to prove that $K_{r}$ lies in a ball $B_{r}$ centered at 0 and with radius $O(r)$, such that $O(r)$ depends continuously on $r$. Hence, if we consider an arbitrary $r_{0}$ and an interval $[c, d]$ containing $r_{0}$, we have that the set $C=\overline{\cup_{c \leq r \leq d} B_{r}}$ is compact and that $\emptyset \neq \omega_{r}(x) \subset C$ for every $x \in \mathbb{R}^{3}$ and every $r$ with $c \leq r \leq d$. Hence the family of Lorenz flows $\varphi_{r}$ is uniformly dissipative for any interval $[c, d]$ of parameters and the corresponding family $\left(K_{r}\right)_{r \in[a, b]}$ of global attractors is a continuation of $K_{r_{0}}$.

From now on we will focus on families of flows in $\mathbb{R}^{n}$. An interesting fact, of topological and dynamical significance, takes place for coercive families. This result provides a graphic characterization of coercive families.

Theorem 11. Let $\varphi_{\lambda}$, with $\lambda \in[0,1]$, be a coercive family of dissipative flows in $\mathbb{R}^{n}$. We denote by $K_{0}$ the global attractor of $\varphi_{0}$ and by $K_{\lambda}$ a continuation of $K_{0}$. Then there exists $\lambda_{0}>0$ such that for every $\lambda$ with $0<\lambda<\lambda_{0}$ there is an isolated invariant compactum $C_{\lambda}$ in $\mathbb{R}^{n} \backslash K_{\lambda}$ such that

i) $C_{\lambda}$ separates $\mathbb{R}^{n}$ into two components and $K_{\lambda}$ lies in the bounded component.

ii) $C_{\lambda}$ has the shape of $S^{n-1}$

iii) $C_{\lambda}$ attracts uniformly all the points of the unbounded component and repels all the points of the bounded one which are not in $K_{\lambda}$.

iv) $\operatorname{diam}\left(C_{\lambda}\right) \rightarrow \infty$ when $\lambda \rightarrow 0$, where $\operatorname{diam}\left(C_{\lambda}\right)$ denotes the diameter of $C_{\lambda}$.

Moreover, the existence of such a $C_{\lambda}$ for $0<\lambda<\lambda_{0}$ is sufficient for the family to be coercive.

Proof. Suppose that $\varphi_{\lambda}$ is a coercive family of flows in $\mathbb{R}^{n}$. Then, if we extend this family to $\mathbb{R}^{n} \cup\{\infty\}$ leaving $\infty$ fixed, there are no connecting orbits between the attractor $K_{\lambda}$ and $\{\infty\}$ when $0<\lambda<\lambda_{0}$ and, as a consequence, $\mathcal{A}_{\lambda}\left(K_{\lambda}\right)$ and $\mathcal{R}_{\lambda}(\{\infty\})$ are disjoint open sets. Besides, $\mathcal{R}_{\lambda}(\{\infty\})$ is connected by [17, Theorem 3.6] and, since there exists $0<\lambda_{1} \leq 1$ (we may assume that $\lambda_{1}=\lambda_{0}$ ) such that $K_{\lambda}$ is connected, [17, Theorem 3.6] also ensures that $\mathcal{A}_{\lambda}\left(K_{\lambda}\right)$ is connected for $0<\lambda_{1} \leq 1$. Since $\mathbb{R}^{n} \cup\{\infty\}$ is connected, then

$$
C_{\lambda}=\mathbb{R}^{n} \backslash\left(\mathcal{A}_{\lambda}\left(K_{\lambda}\right) \cup \mathcal{R}_{\lambda}(\{\infty\})\right)
$$

is non-empty, compact and invariant. It is clear that it is also isolated since every point near $C_{\lambda}$ must be either in $\mathcal{A}_{\lambda}\left(K_{\lambda}\right)$ or in $\mathcal{R}_{\lambda}(\{\infty\})$. Notice that from the previous discussion it also follows that $C_{\lambda}$ decomposes $\mathbb{R}^{n}$ into two connected components and that $K_{\lambda}$ is contained in the bounded one. Besides, it is clear that $C_{\lambda}$ must attract every point in $\mathcal{R}_{\lambda}(\{\infty\})$ (the unbounded component) and repel every point in $\mathcal{A}_{\lambda}\left(K_{\lambda}\right)$ (the bounded one) which is not in $K_{\lambda}$. Now, we will see that $\mathbb{R}^{n} \backslash K_{\lambda}$ has the homotopy type of $S^{n-1}$ and that the inclusion $i_{\lambda}: C_{\lambda} \hookrightarrow \mathbb{R}^{n} \backslash K_{\lambda}$ is a shape equivalence. To see that choose a closed ball $B$ isolating $K_{0}$. Then, for small values of the parameter $B$ is an isolating neighborhood of $K_{\lambda}$ as well, and it is contained in $\mathcal{A}_{\lambda}\left(K_{\lambda}\right)$ (in fact, we may assume that this happens for $0<\lambda<\lambda_{0}$ ). As a consequence $K_{\lambda}$ has a basis of neighborhoods consisting of balls (obtained as images of $B$ by the flow $\varphi_{\lambda}$ ), i.e., it is cellular. Then, by [7, Exercise 2, pg. 41] $\mathbb{R}^{n} \backslash K_{\lambda}$ is homeomorphic to $\mathbb{R}^{n} \backslash\{p\}$, where $p \in \mathbb{R}^{n}$, and hence it has the homotopy type of $S^{n-1}$.

Let us see that $i_{\lambda}: C_{\lambda} \hookrightarrow \mathbb{R}^{n} \backslash K_{\lambda}$ is a shape equivalence. To do that notice that, since $K_{\lambda}$ is an attractor for $0<\lambda<\lambda_{0}$, it possesses a Lyapunov function $\Phi_{\lambda}: \overline{\mathcal{A}_{\lambda}\left(K_{\lambda}\right)} \rightarrow[0, \infty]$ which is strictly decreasing on orbits of $\varphi_{\lambda}$ contained in $\mathcal{A}_{\lambda}\left(K_{\lambda}\right) \backslash K_{\lambda}$ and such that $\left.\Phi_{\lambda}\right|_{K_{\lambda}}=0$ and $\left.\Phi_{\lambda}\right|_{\partial \mathcal{A}_{\lambda}\left(K_{\lambda}\right)}=\infty$. Similarly, since $\{\infty\}$ is a repeller for every $\lambda$, there exist a Lyapunov function $\Psi_{\lambda}: \overline{\mathcal{R}_{\lambda}(\{\infty\})} \rightarrow[0, \infty]$ such that $\Psi_{\lambda}$ is strictly increasing on orbits of $\varphi_{\lambda}$ contained in $\mathcal{R}_{\lambda}(\{\infty\}) \backslash\{\infty\}, \Psi_{\lambda}(\infty)=0$ and $\left.\Psi_{\lambda}\right|_{\partial \mathcal{R}_{\lambda}(\{\infty\})}=\infty$. Using this fact we define, for each $0<\lambda<\lambda_{0}$ a function $F_{\lambda}: \mathbb{R}^{n} \cup\{\infty\} \rightarrow[0, \infty]$ by 


$$
F_{\lambda}(x)= \begin{cases}\Phi_{\lambda}(x) & \text { if } x \in \mathcal{A}\left(K_{\lambda}\right) \\ \Psi_{\lambda}(x) & \text { if } x \in \mathcal{R}(\{\infty\}) \\ 0 & \text { if } x \in K_{\lambda} \cup\{\infty\} \\ \infty & \text { otherwise. }\end{cases}
$$

It is clear that $F_{\lambda}$ is continuous for each $0<\lambda<\lambda_{0}$ and

$$
C_{\lambda}=F_{\lambda}^{-1}(\infty)
$$

For every $a>0$ the set $N_{a}^{\lambda}=F_{\lambda}^{-1}[a, \infty]$ is a compact neighborhood of $C_{\lambda}$. On the other hand, for each $x \in \mathbb{R}^{n} \backslash\left(K_{\lambda} \cup \stackrel{\circ}{N}_{a}^{\lambda}\right)$ there exists a unique time $t_{a}^{\lambda}(x) \in \mathbb{R}$ such that $F_{\lambda}\left(\varphi_{\lambda}\left(x, t_{a}^{\lambda}(x)\right)\right)=a$ and for each $0<\lambda<\lambda_{0}$ the function $t_{a}^{\lambda}: \mathbb{R}^{n} \backslash\left(K_{\lambda} \cup \stackrel{\circ}{N}_{a}^{\lambda}\right) \rightarrow \mathbb{R}$ is continuous. Using this fact we can define the retraction $r_{a}^{\lambda}: \mathbb{R}^{n} \backslash K_{\lambda} \rightarrow N_{a}^{\lambda}$ as follows:

$$
r_{a}^{\lambda}(x)= \begin{cases}\varphi_{\lambda}\left(x, t_{a}^{\lambda}(x)\right) & \text { if } x \in \mathbb{R}^{n} \backslash\left(K_{\lambda} \cup N_{a}^{\lambda}\right) \\ x & \text { if } x \in N_{a}^{\lambda} .\end{cases}
$$

It is straightforward to see that $r_{a}^{\lambda}$ is a deformation retraction from $\mathbb{R}^{n} \backslash K_{\lambda}$ onto $N_{a}^{\lambda}$. Besides, if $a^{\prime}>a$ it is also easy to see that $\left.r_{a^{\prime}}^{\lambda}\right|_{N_{a}^{\lambda}}: N_{a}^{\lambda} \rightarrow N_{a^{\prime}}^{\lambda}$ is a deformation retraction as well. Since the family $\left\{N_{a}^{\lambda}\right\}_{a>0}$ is a basis of neighborhoods of $C_{\lambda}$ it follows that the inclusion $i_{\lambda}: C_{\lambda} \hookrightarrow \mathbb{R}^{n} \backslash K_{\lambda}$ is a shape equivalence.

Moreover, for each $0<\lambda<\lambda_{0}$, the invariant compactum $C_{\lambda}$ contains the compact set $\partial_{\mathcal{A}_{\lambda}}\left(K_{\lambda}\right)$ and, as a consequence, $\operatorname{diam}\left(C_{\lambda}\right) \geq \operatorname{diam}\left(\partial \mathcal{A}_{\lambda}\left(K_{\lambda}\right)\right)=\operatorname{diam}\left(\overline{\mathcal{A}_{\lambda}\left(K_{\lambda}\right)}\right)$. Besides, since $K_{0}$ is a global attractor it follows that $\operatorname{diam}\left(\overline{\mathcal{A}_{\lambda}\left(K_{\lambda}\right)}\right)$ must go to infinity when $\lambda$ goes to zero. Therefore, $\operatorname{diam}\left(C_{\lambda}\right) \rightarrow \infty$ when $\lambda \rightarrow 0$.

Conversely, assume now that there exists such a $C_{\lambda}$. Since $C_{\lambda}$ is an isolated invariant compactum which decomposes $\mathbb{R}^{n}$ into two components with $K_{\lambda}$ lying in the bounded one it follows that $\mathcal{A}_{\lambda}\left(K_{\lambda}\right)$ is also contained in the bounded component.

\section{The general case}

In view of the previous results, it is interesting to study in all its generality the mechanism which produces the bifurcation global to non-global in families of dissipative flows. With this aim we introduce the following definition.

Definition 12. Let $\varphi_{\lambda}: \mathbb{R}^{n} \times \mathbb{R} \rightarrow \mathbb{R}^{n}$, with $\lambda \in[0,1]$, be a parametrized family of dissipative flows. The family is said to be polar if it has arbitrarily large bounded trajectories. More precisely: for every $L>0$ (arbitrarily large) there is a $\lambda_{0}>0$ such that for every $0<\lambda<\lambda_{0}$ there is a bounded trajectory $\gamma_{\lambda}$ of $\varphi_{\lambda}$ and a $t_{\lambda}<0$ such that $\left\|\gamma_{\lambda}(t)\right\|>L$ for every $t$ with $-\infty<t<t_{\lambda}$.

Remark 13. Obviously, if $K_{\lambda}$ is a continuation of the global attractor $K_{0}$ of $\varphi_{0}$, for $L$ sufficiently large, $\gamma_{\lambda}$ lies in $\mathbb{R}^{n} \backslash K_{\lambda}$.

The following proposition makes it clear that polarity is a key notion regarding the transition global to non-global.

Proposition 14. Let $\varphi_{\lambda}: \mathbb{R}^{n} \times \mathbb{R} \rightarrow \mathbb{R}^{n}$, with $\lambda \in[0,1]$, be a parametrized family of dissipative flows. Then the family is polar if and only if for every continuation $K_{\lambda}$ of the global attractor $K_{0}$ of $\varphi_{0}$ there exists $\lambda_{0}$ such that $K_{\lambda}$ is not a global attractor for every $0<\lambda<\lambda_{0}$. 
Proof. If $\varphi_{\lambda}$ is polar then, by Remark 13, for every $\lambda$ with $0<\lambda<\lambda_{0}$ there is a bounded trajectory $\gamma_{\lambda}$ of $\varphi_{\lambda}$ contained in $\mathbb{R}^{n} \backslash K_{\lambda}$ and, thus, $K_{\lambda}$ is not a global attractor. Conversely, if $K_{\lambda}$ is not a global attractor for every $\lambda$ with $0<\lambda<\lambda_{0}$, then there exists at least a bounded orbit $\gamma_{\lambda}$ in $\mathcal{A}_{\lambda}\left(K_{\lambda}\right) \backslash K_{\lambda}$. The negative omega-limit of $\gamma_{\lambda}$ must be contained in $\partial \mathcal{A}_{\lambda}\left(K_{\lambda}\right)$, which is a closed set all whose points are arbitrarily close to $\infty$ when $\lambda \rightarrow 0$. This implies that the orbits $\gamma_{\lambda}$ satisfy the requirements of the definition of polarity when $\lambda_{0}$ is sufficiently small.

The following result describes the overall picture of polar families of dissipative flows.

Theorem 15. If $\varphi_{\lambda}: \mathbb{R}^{n} \times \mathbb{R} \rightarrow \mathbb{R}^{n}$, with $\lambda \in[0,1]$, is a polar family of dissipative flows then there exists $\lambda_{0}>0$ such that for every $\lambda$ with $0<\lambda<\lambda_{0}$ the maximal invariant compactum lying in $\mathbb{R}^{n} \backslash K_{\lambda}$ for the flow $\varphi_{\lambda}$, which we denote by $C_{\lambda}$, is non-empty, isolated and its cohomological Conley index is trivial in every dimension. Moreover, the family $\varphi_{\lambda}$ is coercive if and only if $C_{\lambda}$ has the shape of $S^{n-1}$.

Proof. Let $K_{\lambda}$ be a continuation of the global attractor of $\varphi_{0}$. Since $\varphi_{\lambda}$ is dissipative there exists a global attractor $A_{\lambda}$ of $\varphi_{\lambda}$ and, since $\varphi_{\lambda}$ is polar, there exists $\lambda_{0}>0$ such that $K_{\lambda}$ is a connected attractor and $K_{\lambda} \varsubsetneqq A_{\lambda}$ for $0<\lambda<\lambda_{0}$.

Since $K_{\lambda}$ is an attractor of $\varphi_{\lambda}$, it must be also an attractor of $\left.\varphi_{\lambda}\right|_{A_{\lambda}}$. Let $C_{\lambda}$ be the complementary repeller of $K_{\lambda}$ for $\left.\varphi_{\lambda}\right|_{A_{\lambda}}$. It is clear that $C_{\lambda}$ is a non-empty and invariant compactum. Moreover, if $x$ is a point in $\mathbb{R}^{n} \backslash K_{\lambda}$ not belonging to $C_{\lambda}$ then the trajectory of $x$ either approaches $\infty$ for negative times (if $x \in \mathbb{R}^{n} \backslash A_{\lambda}$ ) or approaches $K_{\lambda}$ for positive times (if $x \in A_{\lambda}$ ). Both situations prevent $x$ from lying in a compact invariant set contained in $\mathbb{R}^{n} \backslash K_{\lambda}$ and, thus, $C_{\lambda}$ is the maximal invariant compactum lying in $\mathbb{R}^{n} \backslash K_{\lambda}$. The same argument proves that $C_{\lambda}$ is isolated.

Now we will see that the cohomological Conley index $C H^{*}\left(C_{\lambda}\right)=0$. Consider the family of flows extended to $\mathbb{R}^{n} \cup\{\infty\}$ leaving $\infty$ fixed. Let $N$ be a positively invariant isolating block of $K_{\lambda}$ such that $N \cap C_{\lambda}=\emptyset$ and let $N_{\infty}$ be a negatively invariant isolating block of $\{\infty\}$ with $N_{\infty} \cap C_{\lambda}=\emptyset$. Then, the compactum $\hat{N}=\mathbb{R}^{n} \backslash\left(\stackrel{\circ}{N} \cup \stackrel{\circ}{N}_{\infty}\right)$ is an isolating block of $C_{\lambda}$ whose exit set $\hat{N}^{o}$ agrees with $\partial N$ and its entrance set $\hat{N}^{i}$ agrees with $\partial N_{\infty}$. Moreover, it is easy to see that $\hat{N} / \partial N$ and $\hat{N} / \partial N_{\infty}$ are connected. This implies that $C H^{0}\left(C_{\lambda}\right)=0$ and that $C H_{0}^{-}\left(C_{\lambda}\right)=0$, where $C H_{*}^{-}\left(C_{\lambda}\right)$ is the homological Conley index for the reverse flow. Then, by the time duality of the Conley index [21] we also have that $C H^{n}\left(C_{\lambda}\right)=0$.

We study now the Morse-Conley sequence of cohomology of the attractor- repeller decomposition $\left(K_{\lambda}, C_{\lambda}\right)$ of $A_{\lambda}[10]$

$$
\cdots \stackrel{\delta}{\rightarrow} C H^{k}\left(C_{\lambda}\right) \rightarrow C H^{k}\left(A_{\lambda}\right) \rightarrow C H^{k}\left(K_{\lambda}\right) \stackrel{\delta}{\rightarrow} \cdots
$$

Since $A_{\lambda}$ is a global attractor it follows from [32, Lemma 2] that $C H^{k}\left(A_{\lambda}\right)=0$ for every $k>0$ and $C H^{0}\left(A_{\lambda}\right)=\mathbb{Z}$. On the other hand, since $K_{\lambda}$ is a continuation of $A_{0}$ and the Conley index is preserved by continuation we also have that $C H^{k}\left(K_{\lambda}\right)=0$ for every $k>0$ and that $C H^{0}\left(K_{\lambda}\right)=\mathbb{Z}$. We deduce from this that $C H^{k}\left(C_{\lambda}\right)=0$ as well for $k>1$. Since we already know that $C H^{0}\left(C_{\lambda}\right)=C H^{n}\left(C_{\lambda}\right)=0$ it remains only to prove that $C H^{1}\left(C_{\lambda}\right)$ is also trivial.

Consider the initial segment of the Morse-Conley sequence

$$
0 \rightarrow C H^{0}\left(C_{\lambda}\right) \rightarrow C H^{0}\left(A_{\lambda}\right) \rightarrow C H^{0}\left(K_{\lambda}\right) \rightarrow C H^{1}\left(C_{\lambda}\right) \rightarrow C H^{1}\left(A_{\lambda}\right) \rightarrow \cdots
$$

Then, the connectedness of $A_{\lambda}$ and $K_{\lambda}$ ensure that the homomorphism

$$
\mathbb{Z} \cong C H^{0}\left(A_{\lambda}\right) \rightarrow C H^{0}\left(K_{\lambda}\right) \cong \mathbb{Z}
$$

is an isomorphism. Therefore, since $C H^{1}\left(A_{\lambda}\right)=0$, it follows that $C H^{1}\left(C_{\lambda}\right)=0$. 
The last part of the theorem follows easily from Theorem 11.

Remark 16. If we only assume that the family $\varphi_{\lambda}$ is not uniformly dissipative then we have an analogous proposition but replacing "there exists $\lambda_{0}$ such that for every $0<\lambda<\lambda_{0}$ " by "for every $\lambda_{0}$ there exists $0<\lambda<\lambda_{0} "$

\section{Acknowledgements}

Some of the results of this paper were obtained when the first author was visiting Jagiellonian University in Krakow. He wishes to express his gratitude to the Mathematics Department of Jagiellonian University and, very especially, to Klaudiusz Wójcik for his hospitality.

\section{References}

[1] N.P. Bhatia, G.P. Szegö, Stability Theory of Dynamical Systems, Classics in Mathematics, Springer, 2002.

[2] K. Borsuk, Theory of Shape, Monografie Matematyczne, vol. 59, Polish Scientific Publishers, Warsaw, 1975.

[3] G. Butler, P. Waltman, Persistence in dynamical systems, J. Differ. Equ. 63 (1986) 255-263.

[4] C. Conley, Isolated Invariant Sets and the Morse Index, CBMS Regional Conference Series in Mathematics, vol. 38, American Mathematical Society, Providence, RI, 1978.

[5] C. Conley, R.W. Easton, Isolated invariant sets and isolating blocks, Trans. Am. Math. Soc. 158 (1971) 35-61.

[6] C. Conley, E. Zehnder, Morse-type index theory for flows and periodic solutions for Hamiltonian equations, Commun. Pure Appl. Math. 37 (1984) 207-253.

[7] R.J. Daverman, Decompositions of Manifolds, Pure and Applied Mathematics, vol. 124, Academic Press, 1986.

[8] J. Dydak, J. Segal, Shape Theory. An Introduction, Lecture Notes in Mathematics, vol. 688, Springer, 1978.

[9] R.W. Easton, Isolating blocks and symbolic dynamics, J. Differ. Equ. 17 (1975) 96-118.

[10] R. Franzosa, Index filtrations and the homology index braid for partially ordered Morse decompositions, Trans. Am. Math. Soc. 298 (1) (1986) 193-213.

[11] B.M. Garay, Uniform persistence and chain recurrence, J. Math. Anal. Appl. 139 (1989) 372-381.

[12] A. Giraldo, M.A. Morón, F.R. Ruiz del Portal, J.M.R. Sanjurjo, Some duality properties of non-saddle sets, Topol. Appl. $113(1-3)$ (2001) 51-59.

[13] A. Giraldo, M.A. Morón, F.R. Ruiz del Portal, J.M.R. Sanjurjo, Shape of global attractors in topological spaces, Nonlinear Anal. 60 (5) (2005) 837-847.

[14] J.K. Hale, Stability and gradient dynamical systems, Rev. Mat. Complut. 17 (1) (2004) 7-57.

[15] A. Hatcher, Algebraic Topology, Cambridge University Press, Cambridge, 2002.

[16] J. Hofbauer, A unified approach to persistence, Acta Appl. Math. 14 (1989) 11-22.

[17] L. Kapitanski, I. Rodnianski, Shape and Morse theory of attractors, Commun. Pure Appl. Math. 53 (2000) $218-242$.

[18] N. Levinson, Transformation theory of non-linear differential equations of the second order, Ann. Math. 45 (2) (1944) $723-737$.

[19] E.N. Lorenz, Deterministic nonperiodic flow, J. Atmos. Sci. 20 (1963) 130-141.

[20] S. Mardešić, J. Segal, Shape Theory. The Inverse System Approach, North-Holland Mathematical Library, vol. 26, NorthHolland Publishing Co., 1982.

[21] M. Mrozek, R. Srzednicki, On time-duality of the Conley index, Results Math. 24 (1993) 161-167.

[22] J. Palis, W. de Melo, Geometric Theory of Dynamical Systems. An Introduction, Springer-Verlag, New York-Berlin, 1982.

[23] S.Y. Pilyugin, Introduction to Structurably Stable Systems of Differential Equations, Birkhaüser Verlag, 1992.

[24] V.A. Pliss, Nonlocal Problems of the Theory of Oscillations, Academic Press, New York, 1966.

[25] J.W. Robbin, D. Salamon, Dynamical systems, shape theory and the Conley index, in: Charles Conley Memorial Volume, Ergod. Theory Dyn. Syst. 8* (1988) 375-393.

[26] J.C. Robinson, Global attractors: topology and finite-dimensional dynamics, J. Dyn. Differ. Equ. 11 (3) (1999) 557-581.

[27] J.C. Robinson, Infinite-Dimensional Dynamical Systems. An Introduction to Dissipative Parabolic PDEs and the Theory of Global Attractors, Cambridge Texts in Applied Mathematics, Cambridge University Press, Cambridge, 2001.

[28] D. Salamon, Connected simple systems and the Conley index of isolated invariant sets, Trans. Am. Math. Soc. 291 (1985) $1-41$.

[29] J.J. Sánchez-Gabites, Dynamical systems and shapes, Rev. R. Acad. Cienc. Exactas Fís. Nat., Ser. A Mat. 102 (1) (2008) $127-159$.

[30] J.M.R. Sanjurjo, An intrinsic description of shape, Trans. Am. Math. Soc. 329 (1992) 625-636.

[31] J.M.R. Sanjurjo, Multihomotopy, Cech spaces of loops and shape groups, Proc. Lond. Math. Soc. 69 (1994) 330-344.

[32] J.M.R. Sanjurjo, On the structure of uniform attractors, J. Math. Anal. Appl. 192 (1995) 519-528.

[33] J.M.R. Sanjurjo, Stability, attraction and shape: a topological study of flows, in: Topol. Methods Nonlinear Anal., Juliusz Schauder Cent. Nonlinear Stud., Toruń, in: Lect. Notes Nonlinear Anal., vol. 12, 2011, pp. 93-122.

[34] J.M.R. Sanjurjo, On the fine structure of the global attractor of a uniformly persistent flow, J. Differ. Equ. 252 (2012) 4886-4897.

[35] E. Spanier, Algebraic Topology, McGraw-Hill, New York, 1966.

[36] C. Sparrow, The Lorenz Equations: Bifurcations, Chaos and Strange Attractors, Springer, Berlin, 1982. 